\title{
Health Is Health Is Health? Age Differences in Intraindividual Variability and in Within-Person Versus Between-Person Factor Structures of Self-Reported Health Complaints
}

\author{
Julia K. Wolff \\ Max Planck Institute for Human Development, Berlin, Germany \\ and German Center of Gerontology, Berlin, Germany \\ Martin Lövdén \\ Max Planck Institute for Human Development, Berlin, Germany \\ and Lund University \\ Ulman Lindenberger \\ Max Planck Institute for Human Development, Berlin, Germany
}

\author{
Annette Brose \\ Max Planck Institute for Human Development, Berlin, Germany
}

Clemens Tesch-Römer

German Centre of Gerontology, Berlin, Germany

Florian Schmiedek

Max Planck Institute for Human Development, Berlin, Germany and German Institute for International Educational Research, Frankfurt am Main, Germany

\begin{abstract}
The variability of health complaints within individuals across time has rarely been studied, and the question whether between- and within-person factor structures of health-related variables are equivalent has not been tested so far. We examined self-reported health complaints in 101 younger (20-31 years) and 103 older adults (65-80 years) over a period of 100 daily assessments. Data were analyzed with confirmatory two-level factor analysis. One-factor structures of health complaints provided an acceptable fit at the between- and average within-person levels in both age groups, supporting the assumption of equivalent average within- and between-person factor structures for health complaints. Age differences in loading patterns indicated that subjective health may be experienced differently by younger and older adults. Small age differences in mean levels of health symptoms were observed. Intraindividual variability in health complaints was reliable. Older adults fluctuated less from day to day than younger adults, presumably reflecting less fluctuation in objective health, differences in response styles, situational influences, or habituation processes. We conclude that future research should consider intraindividual variability as being descriptive of a person's health status, and take possible differences between withinand between-person factor structures of subjective health into account.
\end{abstract}

Keywords: aging, intraindividual variability, within-person factor structure, subjective health

Subjective health, the individual evaluation of one's own health status, is highly important in old age. In the older segments of the population almost everyone has some objectively measurable health problem (e.g., Aldwin, Park, \& Spiro, III, 2007). Despite similar objective diagnoses, subjective health may nonetheless vary substantially between individuals. It has been shown that subjective health is an effective predictor of functional status and longevity, above and beyond objective health status (Benyamini \& Idler, 1999; Idler \& Benyamini, 1997). Developmental research is interested in the change of subjective health over the life span. Even though older adults report lower levels than younger adults, the age differences in subjective health are smaller than age
This article was published Online First July 9, 2012.

Julia K. Wolff, Max Planck Institute for Human Development, Berlin, Germany and German Center of Gerontology, Berlin, Germany; Annette Brose and Ulman Lindenberger, Max Planck Institute for Human Development, Berlin, Germany; Martin Lövdén, Max Planck Institute for Human Development, Berlin, Germany and Department of Psychology, Lund University, Lund, Sweden; Clemens Tesch-Römer, German Center of Gerontology, Berlin, Germany; Florian Schmiedek, Max Planck Institute for Human Development, Berlin, Germany and German Institute for International Educational Research (DIPF), Frankfurt am Main, Germany.

The authors thank Colin Bauer, Gabriele Faust, Katja Müller-Helle, Birgit Heim, Annette Rentz-Lühning, and a team of highly committed student research assistants for their help in conducting the study, and Julia Delius for editorial assistance. The COGITO Study was supported by the Max Planck Society, including a grant from the innovation fund of the Max Planck Society (M.FE.A.BILD0005); the Alexander von Humboldt Foundation's Sofja Kovalevskaja Award (to Martin Lövdén), which is funded by the German Federal Ministry for Education and Research (BMBF); the German Research Foundation (DFG; KFG 163); and the BMBF Center for Advanced Imaging (CAI).

Correspondence concerning this article should be addressed to Julia $\mathrm{K}$. Wolff, who is now at the German Centre of Gerontology, Manfredvon-Richthofen-Str. 2, 12101 Berlin, Germany. E-mail: julia.wolff@ dza.de 
differences in objective health measures (e.g., Staudinger, Fleeson, \& Baltes, 1999; Wurm, Lampert, \& Menning, 2009). Whereas subjective and objective health ratings are relatively congruent in younger ages, they drift apart in older adulthood. This raises the question whether subjective health has different meanings for younger and older adults. Potentially, younger and older adults focus on different aspects when evaluating their own health. Investigating such differences is important for understanding subjective health in both age groups, but also for interpreting potential age-differential relationships to other variables. Therefore, the goal of this study is to examine the nature of subjective health in younger and older adulthood using confirmatory factor analysis.

\section{Intraindividual Change and Intraindividual Variability in Health Complaints}

Central to our approach is a developmental perspective that not only considers long-term changes (taking place over years or decades), but also short-term fluctuations (occurring over hours, days, or weeks). Whereas subjective health status may change slowly over the life course, subjective health complaints may vary with higher volatility, fluctuating from day to day. The kind of health complaints (like headaches or dyspnea) or the severity of complaints may change from moment to moment or from day to day. Therefore, subjective health can be viewed not only as a rather stable, slowly changing characteristic of a person, but also as a construct that fluctuates from one day to the next (Becker, Schulz, \& Scholtz, 2004). These two perspectives cannot be discriminated with a single measurement (Nesselroade, 2001). Intraindividual variability, if present, can only be observed if a construct is measured multiple times in the same person. Such within-person variability is often treated as measurement error, though it may point to important state-like phenomena that uniquely add to the description and prognosis of an individual's health status (Becker et al., 2004; Nesselroade, 1991). Thus, the concept of health should not be approached at the trait level only, but extended to intraindividual variability in health symptoms.

Intraindividual variability in self-reported health complaints is likely to change with increasing age. Day-to-day fluctuations in health symptoms (and their sensitivity to situational influences) can be seen as driven by the objective health status of a person. Older adults' declining objective health status and increasing frailty may be associated with larger fluctuations in subjectively experienced health complaints from one day to the next. In line with this, the risk for cardiovascular diseases is known to be associated with larger fluctuations in blood pressure (e.g., Schwartz, Tuner, Moore, \& Sing, 2000). Likewise, senescent brain changes have been linked to increasing intraindividual fluctuations in cognitive performance (MacDonald, Li, \& Bäckman, 2009). Thus, in the present study, we expect older adults to fluctuate more from day to day than younger adults.

\section{The Structure of Health Complaints: Between- and Within-Person Perspectives}

Within-person associations between health, stress, and wellbeing have increasingly come under scrutiny (for an overview, see Affleck, Tennen, Urrows, \& Higgins, 1994; Charles \& Almeida, 2006; Ong \& Allaire, 2005). Typically, summary scores of differ- ent health items are used to characterize an individual's subjective health. Such composite scores are often empirically supported by factor analyses based on between-person variability, meaning that they can differentiate well between different persons. However, to investigate within-person processes over time, within-person factor structures that differentiate well within individuals over time are also needed. For example, when analyzing the within-person relationship between health and daily well-being, one would like to use a composite score of health that is meaningful, has good psychometric properties, and is either similar across age groups or incorporates age-group differences that are known and therefore interpretable. Hence, from a developmental perspective, the structure of self-reported health should not only be studied at the between-person, but also at the within-person level, taking into account fluctuations within persons over time.

Such an approach can provide various insights. First, an index of intraindividual variability in physical symptoms was previously shown to be meaningful in a study observing older adults' subjective health over 25 weeks, because it proved predictive of mortality (Ghisletta, Nesselroade, Featherman, \& Rowe, 2002). That is, intraindividual variability in physical symptoms may mark systematic individual as well as age-group differences. Second, the structure of interindividual variation may differ from the structure of intraindividual variation. As there are more and more studies on aging that investigate within-person processes by means of momentary assessment (e.g., the relationship between daily events and intensity of chronic pain; Affleck, et al., 1994), a solid understanding of the potentially age-differential nature of constructs at the within-person level is required. Therefore, in this study we do not only investigate age differences in means and intraindividual variability in subjective health complaints, but also in structural aspects by means of confirmatory factor analyses (CFA) at withinand between-person levels.

It is crucial to note, between-person factor structures do not necessarily generalize to the within-person level (Molenaar \& Campbell, 2009). Psychometric properties of scales evaluated with between-person data are also not transferable to scales measuring within-person change. As a consequence, the between-person approach may fail to capture important aspects of health-related processes, as it neglects within-person differences in structure. For example, a measure of health complaints that is validated to assess between-person differences may reflect subjective health in different dimensions and with different psychometric properties when applied to within-person data. Thus, it is necessary to investigate structural aspects of phenomena at both levels, the level of interindividual differences, and the level of intraindividual differences.

The ergodicity or homogeneity assumption postulates that the between-person factor structure of a construct is identical to every within-person factor structure of each individual (Borsboom, Mellenbergh, \& van Heerden, 2003; Molenaar, 2004). Initial evidence indicates that the ergodicity assumption does not hold in the domains of motivation and personality (Brose, Schmiedek, Lövdén, Molenaar, \& Lindenberger, 2010; Hamaker, Nesselroade, \& Molenaar, 2007). Simply assuming equivalence without testing it is troublesome, because the measures used on the within-person level may not measure the construct adequately. This could be associated with low reliability and validity of the measures, and as a consequence, results from within-person data may overlook weaknesses in the used measures. 
One necessary but not sufficient condition for ergodicity is that the between-person and the average within-person factor structures do not differ reliably from each other. If this is not the case, the ergodicity assumption can be rejected without testing the factor structure for each individual. If the between-person and the average within-person structures are comparable, ergodicity is still not verified, as individual within-person structures may deviate from the average within-person structure. Thus, the comparison of average structures represents an important step toward the ergodicity assumption. In the present study, the items used to assess subjective health complaints were partly formulated based on the subscales of the Giessen Subjective Complaints List (Brähler, Hinz, \& Scheer, 2008). In the original version, the subscales can form one overall sum score that is validated on the between-person level and assesses subjective health with the same items across all age groups. Therefore, we expect the health complaints to form one latent factor on the between-person level in younger and older adults. To compare the one-factor structure with the average within-person level and across age groups, we tested the same factor solution on the average within-person level as on the between-person level in both age groups. However, differences in fit indices and loading patterns between levels and age groups may occur. Keeping the incongruence of subjective and objective health in older adults in mind, differences in subjective health within an older adult may be described by other complaints and in other domains than within a younger adult. Thus, in the current study, we explore age and level differences in loading patterns of the estimated factor structures.

Taken together, we examine whether this study replicates previous findings on lower levels of subjective health in older than younger adults. Moreover, we hypothesize that older adults show more intraindividual variability in subjective health complaints. In addition, we tested the invariance of factor solutions on the between-person and the average within-person levels in two age groups in the domain of subjective health and explored level and age differences in fit indices and loading patterns.

\section{Method}

This study is part of a larger study, the COGITO Study, which was set up to systematically explore intraindividual variability in different psychological domains such as cognitive functioning, motivation, affect, and stress reactions (see Brose, Schmiedek, Lövdén, \& Lindenberger, 2011; Schmiedek, Bauer, Lövdén, Brose, \& Lindenberger, 2010; Schmiedek, Lövdén, \& Lindenberger, 2010; Schmiedek, Lövdén, \& Lindenberger, 2009, for details). In COGITO, participants went through 10 days of pretesting, followed by a microlongitudinal phase of an average of 101 days, followed by 10 days of posttesting. The current study focuses on data from a daily questionnaire of the 101 assessments. The procedure and cognitive tasks of the daily sessions are described elsewhere (Schmiedek, Lövdén, \& Lindenberger, 2010).

\section{Participants}

The sample consisted of 204 participants, 101 younger adults (52 women; 20-31 years) and 103 older adults (51 women; $65-80$ years). The participants could choose freely when they wanted to come to the laboratory between $8 \mathrm{a} . \mathrm{m}$. and 8:30 p.m. from Monday to Saturday. They were requested to come at least three times per week. Sixty-one percent of the sessions of the older adults and $30 \%$ of the younger adults' sessions took place between 8 a.m. and 12 p.m. Between 12 p.m. and 4 p.m., $28 \%$ of the older adults and $39 \%$ of the younger adults came to the laboratory. Between 4 p.m. and 9 p.m., $12 \%$ of the older adults' sessions and $31 \%$ of the younger adults' sessions took place. The younger adults attended on average $4.44(S D=0.90)$ sessions per week, the older adults on average $3.62(S D=1.49)$ sessions per week, which represents a significant difference, $t=4.74, p<.0001$. The mean difference in days between two sessions varied significantly between younger and older adults: $M(S D)_{\mathrm{YA}}=1.67(0.47) ; M(S D)_{\mathrm{OA}}=1.85(0.59)$, $t=2.40, p=.02$. However, as one to two days of difference between the sessions are tolerated in the requested three days per week, both age groups seemed to be equally compliant to the protocol. During the microlongitudinal phase of the study, $14 \%$ of the younger adults and $4 \%$ of the older adults dropped out of the sample.

On average, the older adults reported 3.1 diseases, with 93\% reporting one or more. For the younger adults, the average number of reported illnesses was $0.76 ; 41 \%$ were free of diseases. After the study, $90 \%$ of the older adults and $50 \%$ of the younger adults also started to participate in the Socio-Economic Panel (SOEP), a representative household survey in Germany conducted by the German Institute for Economic Research (for details, see Wagner, Frick, \& Schupp, 2007). To evaluate the representativeness of the COGITO sample concerning health, the participants were compared with the members of the Berlin subsample of the SOEP in the same age range on two variables, namely self-rated health ("How would you describe your current health?" rated from $0=$ very good to $4=\mathrm{bad}$ ) and number of visits to a doctor in the past three months. The younger and older COGITO participants reported significantly better self-rated health: Older adults (OA): $M_{\text {SOEP }}=3.35, M_{\text {COGITO }}=2.60, t=7.09, p<.0001, \mathrm{ES}=$ -0.68 ; younger adults (YA): $M_{\text {SOEP }}=2.50, M_{\text {COGITO }}=1.98, t=$ $3.30, p=.001, \mathrm{ES}=-0.56$. However, they showed no significant differences compared with the Berlin subsample of the SOEP regarding the number of visits to a doctor $\left(\mathrm{OA}: M_{S O E P}=4.03\right.$, $M_{\text {COGITO }}=3.26, t=1.41, p=.16, \mathrm{ES}=-0.17$; YA: $M_{\text {SOEP }}=$ $\left.2.72, M_{\text {COGITO }}=2.74, t=0.39, p=.70, \mathrm{ES}=0.01\right)$. The COGITO participants who refused to participate in the SOEP did not differ from the participants with SOEP in health and wellbeing measures. However, due to the low SOEP participation rate of the younger adults, the comparisons of this age group should be regarded with caution.

\section{Measures}

The data used in the current study were collected with the questionnaire administered at the beginning of each of the daily sessions. Four of the assessed health complaints were formulated on basis of subscales of the Giessen Subjective Complaints List, namely headaches and limb aches, gastrointestinal complaints, cardiovascular complaints, and exhaustion (Brähler et al., 2008). In addition, upper respiratory complaints, and symptoms of restlessness were included in the questionnaire. Participants were asked whether they were experiencing one of these complaints on that particular day and rated them on a 4-point Likert scale with 0 (no, not at all) to 3 (yes, very much). 


\section{Data Analysis}

The data from the microlongitudinal part of the COGITO Study have a multilevel structure. Each person was measured across 87 to 109 occasions. Therefore, it is possible to investigate the data not only on a between-person level (e.g., means, between-person factor structures) but also on a within-person level (e.g., intraindividual variability, within-person factor structures).

In order to answer the research questions concerning age differences in mean levels and intraindividual variability in the subjective health complaints, we estimated the mean and the average intraindividual standard deviation in each age group. The size of the age difference was evaluated by calculating effect sizes with Cohen's $d$.

The high number of measurement occasions per person allows the estimation of factor structures at the within-person level using the intraindividual covariance matrices of the items. In addition, the sample size of 103 older and 101 younger adults permits the estimation of between-person factor structures using the interindividual covariance matrix of the items.

In order to answer the research questions about differences in factor structure concerning within-person and between-person levels and concerning the age groups, two-level CFA were conducted with Mplus as suggested by Muthén (1994). This analytical approach first separates the total covariance information into withinand between-person components. This results in an estimated between-person covariance matrix for the individual means and an estimated average within-person covariance matrix that results from pooling the covariance matrices of each individual. The between-person model (based on the between-person covariance matrix) and the average within-person model (based on the average within-person covariance matrix) are estimated simultaneously. In Mplus, it is possible to combine such two-level models with multigroup models, which allows us to constrain loadings to be equal not only across levels, but also across age groups. Figure 1 illustrates the expected multigroup two-level model for the six health complaints. The model is identical for the two age groups. The within-person model corresponds to the conventional latentfactor model representing the observed within-person data. In the between-person model, the latent variable is estimated based on the estimated means (represented as circles). Note that the analyses do not account for temporal dependency in the data.

Calculation of two-level factor analysis requires sufficient between- and within-person variance in all variables. Mplus estimates intraclass correlation coefficients (ICC) for each variable, which represent the ratio of the maximum-likelihood estimates of the latent within- and between-person variance components, that is, the proportion of between-person variance in relation to the total variance (i.e., between-person and within-person variance). The ICC ranges between zero and one. If it is close to zero, the variance consists almost entirely of within-person variance, and a two-level model would not be appropriate for the variable because it consists of almost no between-person variance. Muthén and Satorra (1995) proposed the calculation of a design effect (deff), where deff $=1+(c-1) \times \mathrm{ICC}$, and $c$ is the cluster size on the higher level (i.e., in this case, number of participants). As a rule of thumb, with $\operatorname{deff} \geq 2$, a two-level model is appropriate (Muthén \& Satorra, 1995). The ICC and design effect of each variable for the younger and older adults are shown in Table 1. For older adults,

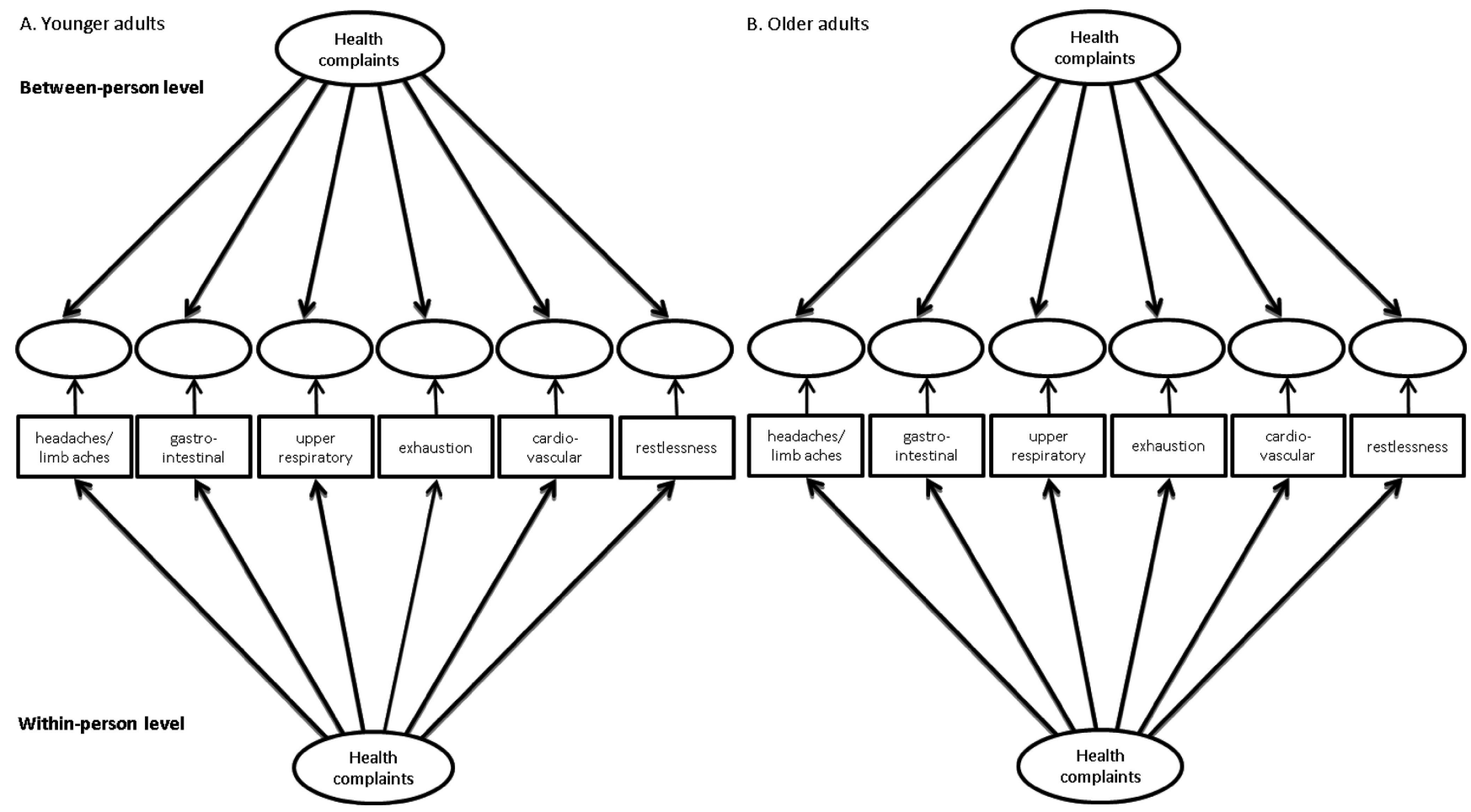

Figure 1. Postulated multigroup, two-level model, with rectangles indicating observed variables and circles denoting latent variables. 
Table 1

Intraclass Correlation Coefficients and Design Effects of Health Complaints by Age Group

\begin{tabular}{|c|c|c|c|c|}
\hline \multirow[b]{2}{*}{ Health complaint } & \multicolumn{2}{|c|}{ Younger adults } & \multicolumn{2}{|c|}{ Older adults } \\
\hline & $\mathrm{ICC}$ & deff & $\mathrm{ICC}$ & deff \\
\hline Gastrointestinal & .18 & 37.54 & .001 & 1.20 \\
\hline Cardiovascular & .37 & 76.11 & .17 & 35.51 \\
\hline Headaches & .25 & 51.75 & .62 & 126.86 \\
\hline Restlessness & .45 & 92.35 & .48 & 98.44 \\
\hline Upper respiratory & .27 & 55.81 & .43 & 88.29 \\
\hline Exhaustion & .31 & 63.93 & .33 & 67.99 \\
\hline
\end{tabular}

Note. $\quad$ ICC $=$ intraclass correlation coefficient; deff $=$ design effect.

the ICC of gastrointestinal complaints was very close to zero, and the design effect was under the limit of deff $=2$. In younger adults, gastrointestinal complaints also had the lowest ICC value. Hence, this variable was excluded from further analyses.

The estimation of a multigroup two-level model with two age groups results in four submodels. The loadings of these submodels can be compared across levels and age groups: An average withinperson model for YA, a between-person model for YA, an average within-person model for OA, and a between-person model for OA. For the investigation of invariance across levels and age groups, equality constraints were made. The following steps were done in order to compare loadings under increasing equality constraints: (a) For each age group, the two-level model was estimated separately with no model constraints, that is, all parameters were freely estimated. (b) Next, the within- and between-person loadings were set equal separately for each age group. (c) A multigroup two-level model with free loadings across levels and age groups was fitted. (d) Only the between-person loadings were set equal across age groups. (e) Only the within-person loadings were set equal across age groups. (f) In a final multigroup two-level model, all loadings were set equal across levels and age groups.

Nested models were compared using the $\chi^{2}$ difference test and the root deterioration per restriction (RDR; Browne \& Du Toit, 1992), with $90 \%$ confidence intervals (CI). The RDR measures the degree to which the constraints affect the model fit. A value of RDR $<.08$ indicates an unsubstantial loss of fit (Browne \& Du Toit, 1992). Exact $p$ values are reported and values $<.05$ are interpreted as significant. ${ }^{1}$

\section{Results}

\section{Age Group Differences in Means}

Means and variances of the health complaints were calculated separately for the two age groups (see Table 2). In line with our predictions, the older adults reported more cardiovascular complaints than the younger adults $(d=-0.33)$. However, younger adults reported more exhaustion $(d=0.63)$ and more upper respiratory complaints $(d=0.34)$ than the older adults.

\section{Age Differences in Intraindividual Variability}

The research question concerning age differences in intraindividual variability was addressed with comparisons of intraindi- vidual standard deviations (ISD), shown in Table 3 . In contrast to our predictions, older adults showed less intraindividual variability than younger adults in all but one health complaint. The effect size of the difference was high for upper respiratory symptoms and for exhaustion $\left(d_{\text {respiratory }}=0.87, d_{\text {exhaustion }}=1.09\right)$. For headaches and limb aches, the effect size was moderate $(d=0.55)$. The effect size of the difference for symptoms of restlessness was small $(d=$ 0.41). Cardiovascular complaint was the only variable for which no reliable mean differences existed.

\section{Confirmatory Factor Analyses With Mplus}

Before answering the research question of equivalent loading patterns across within- and between-person levels and age groups, the fit of the one-factor solution was evaluated separately for the within- and between-person level and for both age groups for the following variables: headaches and limb aches, cardiovascular complaints, exhaustion, upper respiratory complaints, and symptoms of restlessness. The one-factor solution did not fit satisfactorily at the between-person level in either age group: YA: $\chi^{2}=$ $15.51, d f=5$, comparative fit index $(\mathrm{CFI})=.94$, root mean square error of approximation (RMSEA) $=.14,90 \% \mathrm{CI}(.07, .23)$, standardized root mean square residual $(\mathrm{SRMR})=.05$; OA: $\chi^{2}=$ 28.01, $d f=5, \mathrm{CFI}=.85, \mathrm{RMSEA}=.21,90 \%$ CI $(.14, .29)$, SRMR $=.09$. Therefore, residuals were allowed to covary across indicators. For younger adults, the covariance for headaches and limb aches and restlessness, and for older adults the covariance between exhaustion and restlessness, were added. The fits of the resulting modified between-person models were good: YA: $\chi^{2}=$ 4.03, $d f=4, \mathrm{CFI}=1, \mathrm{RMSEA}=.01,90 \% \mathrm{CI}(0, .15), \mathrm{SRMR}=$ .03 ; OA: $\chi^{2}=5.56, d f=4, \mathrm{CFI}=.99, \mathrm{RMSEA}=.06,90 \% \mathrm{CI}$ $(0, .17)$, SRMR $=.03$. For comparison, the one-factor model was also applied to the within-person level and showed a good fit in both age groups: YA: $\chi^{2}=2.16, d f=5, \mathrm{CFI}=1$, RMSEA $=0$, $90 \%$ CI $(0, .08)$, SRMR $=.03$; OA: $\chi^{2}=1.99, d f=5$, CFI $=1$, RMSEA $=0,90 \%$ CI $(0, .08)$ SRMR $=.03)$.

To test for the equivalence of the between-person structures and the average within-person structures, the loadings of the four submodels (average within-person model for YA, between-person model for YA, average within-person model for OA, and betweenperson model for OA) were compared step by step, starting with the equivalence of the average within- and between-person models within the two age groups, and ending with a model in which all loadings across levels and age groups were set equal. The fit indices, including $\chi^{2}$ difference tests and RDR, of all models are shown in Table 4.

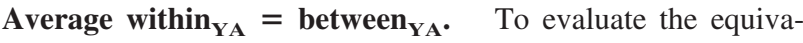
lence of the average within- and between-person factor structures in each age group, the two-level models were estimated separately for younger and older adults. For the younger adults, the models with no constraints (Model 1) was compared to the model with the within- and between-person loadings set to be equal (Model 2). The $\chi^{2}$ difference between the constrained and the unconstrained

\footnotetext{
${ }^{1}$ As the health complaints were not normally distributed, the Mplus analyses were also run with the maximum likelihood estimation method with robust standard errors (MLR) that is robust to nonnormality. This estimation procedure did not change the pattern of results.
} 
Table 2

Means and Standard Deviations of Health Complaints by Age Group

\begin{tabular}{|c|c|c|c|c|c|c|c|}
\hline \multirow[b]{2}{*}{ Health complaint } & \multicolumn{2}{|c|}{ Younger adults } & \multicolumn{2}{|c|}{ Older adults } & \multirow[b]{2}{*}{$t$} & \multirow[b]{2}{*}{$p$} & \multirow[b]{2}{*}{ Effect size } \\
\hline & $M(S D)$ & Range & $M(S D)$ & Range & & & \\
\hline Exhaustion & $0.46(0.44)$ & $0-2.20$ & $0.23(0.28)$ & $0-1.28$ & 4.52 & $<.0001$ & 0.63 \\
\hline Cardiovascular & $0.06(0.18)$ & $0-1.33$ & $0.15(0.34)$ & $0-1.89$ & -2.37 & .02 & -0.33 \\
\hline Headaches & $0.29(0.32)$ & $0-1.33$ & $0.38(0.54)$ & $0-2.48$ & -1.48 & .14 & -0.21 \\
\hline Restlessness & $0.26(0.40)$ & $0-1.80$ & $0.17(0.30)$ & $0-1.68$ & 1.99 & .05 & 0.28 \\
\hline Upper respiratory & $0.34(0.35)$ & $0-1.71$ & $0.23(0.34)$ & $0-1.72$ & 2.42 & .02 & 0.34 \\
\hline
\end{tabular}

model was statistically reliable. At the same time, the RDR indicated that the observed loss in fit was not substantial, $\mathrm{RDR}=.03$;

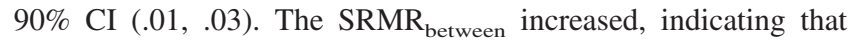
constraining the between- and within-person factor loadings to be equal lowered the fit of the between-person structure more than the fit of the average within-person structure.

Average within $_{\mathbf{O A}}=$ between $_{\mathbf{O A}}$. Similarly, the older adults' model with free loadings across levels (Model 3) was compared to the model with equal loadings across levels (Model 4). The $\chi^{2}$ difference test indicated a significant difference be-

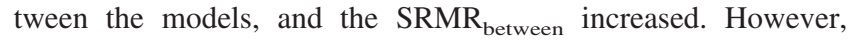
according to the RDR, the loss of fit was not substantial, RDR = $.03 ; 90 \%$ CI $(.03, .05)$.

Between $_{\mathbf{O A}}=$ between $_{\mathbf{Y A}}$. To evaluate the equivalence of the between-person structures across the age groups, the betweenperson loadings of the younger and older adults were constrained to be equal in a multigroup two-level model (Model 6), and were compared to a model with no constraints on the factor loadings (Model 5). The $\chi^{2}$ test indicated a reliable decrease in model fit relative to Model 5, and the RDR indicated that this loss of fit was not substantial, RDR $=.03 ; 90 \%$ CI $(.02, .04)$.

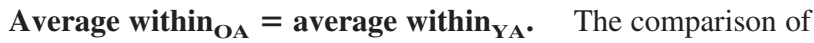
the within-person structures across age groups was tested with Model 7, where the within-person loadings were set to be equal. Here, the $\chi^{2}$ difference test was reliable, and the RDR indicated that the observed loss in fit was substantial, relative to the unconstrained Model 5, RDR $=.08 ; 90 \%$ CI (.07, .09).

Average $_{\text {within }_{\mathrm{OA}}}=$ average within $_{\mathrm{YA}}=$ between $_{\mathrm{OA}}=$ between $_{\mathbf{Y A}}$. In Model 8, a model with all loadings set to be equal across levels and age groups was estimated and compared to the unconstrained model (Model 5). For this comparison, the $\chi^{2}$ difference test was again significant, whereas the RDR indicated a nonsubstantial loss of fit, RDR $=.07 ; 90 \%$ (CI .06, .07), and the $\mathrm{SRMR}_{\text {between }}$ increased.
In sum, the results of model comparisons with the $\chi^{2}$ difference test and RDR showed conflicting results. Whereas the RDR mostly indicated an equivalence of the average withinand between-person loadings, the $\chi^{2}$ difference tests showed reliable differences. To evaluate whether the differences between the submodels can be attributed to individual loadings, in post hoc comparisons, the loadings of the submodels were set equal one by one across levels or age groups, respectively (see Table 5 for the standardized and unstandardized loading patterns of the unconstrained model). The difference of the single loadings was tested for significance with the $\Delta \chi^{2}$ of the constrained versus unconstrained model.

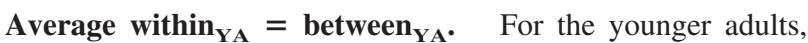
only the loading of restlessness differed significantly between average within-person and between-person level, $\Delta \chi^{2}(1)=18.82$, $p<.0001$. This variable showed a higher loading at the betweenperson level than at the average within-person level.

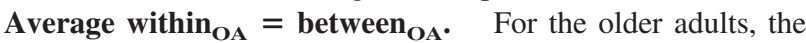
loadings of exhaustion and restlessness had significantly higher loadings at the average within-person than at the between-person level, $\Delta \chi^{2}(1)_{\text {exhaustion }}=26.75, p<.0001 ; \Delta \chi^{2}(1)_{\text {restlessness }}=$ $17.53, p<.0001$.

Between $_{\mathbf{O A}}=$ between $_{\mathbf{Y A}}$. Among the between-person levels, all freely estimated loadings showed significant differences between younger and older adults. Exhaustion, restlessness and respiratory complaints had higher loadings, $\Delta \chi^{2}(1)_{\text {exhaustion }}=$ $31.23, p<.0001 ; \Delta \chi^{2}(1)_{\text {restlessness }}=27.15, p<.0001 ;$ $\left.\Delta \chi^{2}(1)_{\text {respiratory }}=8.23, p=.004\right)$ and cardiovascular complaints had lower loadings in the younger adults than in the older adults, $\Delta \chi^{2}(1)_{\text {cardiovascular }}=5.77, p=.02$. In addition, in the group of older adults, the between-person loadings headaches and limb aches were the highest, whereas exhaustion showed the highest loadings in the other submodels.

Table 3

Intraindividual Standard Deviations of Health Complaints by Age Group

\begin{tabular}{|c|c|c|c|c|c|c|c|}
\hline \multirow[b]{2}{*}{ Health complaint } & \multicolumn{2}{|c|}{ Younger adults } & \multicolumn{2}{|c|}{ Older adults } & \multirow[b]{2}{*}{$t$} & \multirow[b]{2}{*}{$p$} & \multirow[b]{2}{*}{ Effect size } \\
\hline & $M(S D)$ & Range & $M(S D)$ & Range & & & \\
\hline Exhaustion & $0.59(0.25)$ & $0-1.22$ & $0.33(0.21)$ & $0-0.84$ & 7.76 & $<.0001$ & 1.09 \\
\hline Cardiovascular & $0.14(0.18)$ & $0-1.07$ & $0.18(0.22)$ & $0-0.85$ & -1.24 & .22 & -0.17 \\
\hline Headaches & $0.47(0.25)$ & $0-1.23$ & $0.33(0.25)$ & $0-0.91$ & 3.92 & $<.0001$ & 0.55 \\
\hline Restlessness & $0.34(0.29)$ & $0-1.25$ & $0.24(0.20)$ & $0-0.76$ & 2.94 & .003 & 0.41 \\
\hline Upper respiratory & $0.51(0.23)$ & $0-0.99$ & $0.30(0.25)$ & $0-1.08$ & 6.18 & $<.0001$ & 0.87 \\
\hline
\end{tabular}


Table 4

Fit Statistics of Multigroup Two-Level Models

\begin{tabular}{|c|c|c|c|c|c|c|c|c|c|c|c|c|}
\hline \multirow[b]{2}{*}{ Models } & \multirow[b]{2}{*}{ Constraints $^{\mathrm{a}}$} & \multirow[b]{2}{*}{$x^{2}$} & \multirow[b]{2}{*}{$d f$} & \multirow[b]{2}{*}{ CFI } & \multicolumn{2}{|c|}{ RMSEA } & \multirow[b]{2}{*}{$\mathrm{SRMR}_{\mathrm{w}}$} & \multirow[b]{2}{*}{$\mathrm{SRMR}_{\mathrm{b}}$} & \multirow[b]{2}{*}{ Compared with } & \multirow[b]{2}{*}{$p$} & \multirow[b]{2}{*}{ RDR } & \multirow[b]{2}{*}{ Observed power } \\
\hline & & & & & Value & $90 \% \mathrm{CI}$ & & & & & & \\
\hline \multicolumn{13}{|c|}{ Younger adults } \\
\hline Model 1 & none & 217.8 & 9 & .94 & .05 & [.04-.07] & .03 & .03 & & & & \\
\hline Model 2 & $\mathrm{~b}=\mathrm{w}$ & 248.1 & 13 & .93 & .04 & {$[.04-.06]$} & .03 & .13 & Model 1 & .00 & 0.03 & .97 \\
\hline \multicolumn{13}{|l|}{ Older adults } \\
\hline Model 3 & none & 206.5 & 9 & .92 & .05 & {$[.04-.07]$} & .03 & .03 & & & & \\
\hline Model 4 & $\mathrm{~b}=\mathrm{w}$ & 245.4 & 13 & .90 & .04 & {$[.04-.06]$} & .04 & .18 & Model 3 & .00 & 0.03 & .99 \\
\hline \multicolumn{13}{|l|}{ Multigroup } \\
\hline Model 5 & none & 340.3 & 22 & .94 & .04 & {$[.03-.04]$} & .03 & .05 & & & & \\
\hline Model 6 & $\mathrm{~b}_{\mathrm{y}}=\mathrm{b}_{\mathrm{o}}$ & 387.6 & 26 & .93 & .04 & {$[.03-.04]$} & .03 & .15 & Model 5 & .00 & 0.03 & .99 \\
\hline Model 7 & $\mathrm{w}_{\mathrm{y}}=\mathrm{w}_{\mathrm{o}}$ & 623.3 & 26 & .88 & .05 & {$[.04-.05]$} & .04 & .05 & Model 5 & .00 & 0.08 & 1.00 \\
\hline Model 8 & $b_{y}=b_{o}=w_{y}=w_{o}$ & 694.2 & 34 & .87 & .04 & {$[.04-.05]$} & .04 & .17 & Model 5 & .00 & 0.07 & 1.00 \\
\hline
\end{tabular}

Note. $\quad d f=$ degrees of freedom; $\mathrm{CFI}=$ comparative fit index; RMSEA $=$ root mean square of error approximation; $\mathrm{SRMR}_{\mathrm{w}}=$ standardized root mean squared residual for the within-person model; $\mathrm{SRMR}_{\mathrm{b}}=$ standardized root mean squared residual for the between-person model; RDR $=$ root deterioration per restriction.

${ }^{\mathrm{a}}$ Column 2 shows which of the submodels were constrained by equal loadings; $\mathrm{y}=$ younger adults; $\mathrm{o}=$ older adults; $\mathrm{w}=$ within-person model; $\mathrm{b}=$ between-person model.

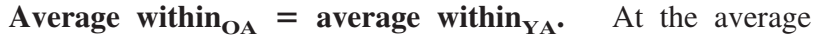
within-person level, the loadings of cardiovascular complaints and restlessness were significantly higher, and the loading of exhaustion was significantly lower in the older adults than in the younger adults, $\Delta \chi^{2}(1)_{\text {exhaustion }}=29.23, p<.0001 ; \Delta \chi^{2}(1)_{\text {cardiovascular }}=$ 97.24, $p<.0001 ; \Delta \chi^{2}(1)_{\text {restlessness }}=42.15, p<.0001$.

These analyses show the differences in the loading patterns in more detail. Overall, differences between age groups were more pronounced than differences between between-person and average within-person analyses within age groups, especially in relation to the exhaustion and cardiovascular complaints variables.

\section{Follow-Up Analyses}

As the individual factor structures are not necessarily captured in the average within-person structure, we also explored in follow-up analyses whether the one-factor solution provides a satisfactory rendition of the data at the individual level. Because of the multiple occasions per individual, it is possible to estimate a confirmatory factor structure for each individual using the SAS code, PROC CALIS. Note that this approach does not account for temporal dependencies in the data, and is therefore comparable to the average within-person structure estimated with Mplus. We computed CFAs using the same one-factor solution as the average within-person factor structure for each person including the five complaints: cardiovascular complaints, headaches and limb aches, exhaustion, respiratory complaints, and restlessness. The loadings were allowed to vary between individuals. The models were estimated for 44 younger and for 37 older adults. The other individuals showed no variance on one or more of the complaints $(n=116)$, or the model did not converge $(n=7)$. To evaluate the models at the level of the subsamples, the average RMSEA of all individual RMSEAs was calculated, separately for the age groups (YA: average RMSEA $=.10, S D=.09$, range $=.00-.28$; OA: average RMSEA $=.08, S D=.09$, range $=.00-.31)$. Of the 44 younger and 37 older adults whose data could be fitted to the one-factor

Table 5

Factor Loading Pattern of Multi-Group Two-Level Model Without Constraints

\begin{tabular}{|c|c|c|c|c|c|}
\hline \multirow[b]{2}{*}{ Model } & \multicolumn{5}{|c|}{ Complaint $^{\mathrm{a}}$} \\
\hline & Exhaustion & Cardiovascular & Headaches & Restlessness & Upper respiratory \\
\hline \multicolumn{6}{|l|}{ Younger adults } \\
\hline Unstandardized within & $1.62(.07)$ & $0.29(.01)$ & 1.00 (n.a.) & $0.59(.03)$ & $0.78(.03)$ \\
\hline Standardized within & $.64(.01)$ & $.32(.01)$ & $.48(.01)$ & $.34(.01)$ & $.36(.01)$ \\
\hline Unstandardized between & $1.46(.20)$ & $0.32(.07)$ & 1.00 (n.a.) & $1.29(.20)$ & $0.85(.14)$ \\
\hline Standardized between & $.82(.05)$ & $.50(.09)$ & $.84(.06)$ & $.84(.05)$ & $.63(.07)$ \\
\hline \multicolumn{6}{|l|}{ Older adults } \\
\hline Unstandardized within & $1.11(.06)$ & $0.62(.04)$ & 1.00 (n.a.) & $1.10(.08)$ & $0.69(.04)$ \\
\hline Standardized within & $.50(.02)$ & $.39(.02)$ & $.42(.02)$ & $.24(.01)$ & $.31(.02)$ \\
\hline Unstandardized between & $0.30(.08)$ & $0.61(.11)$ & 1.00 (n.a.) & $0.34(.08)$ & $0.39(.09)$ \\
\hline Standardized between & $.48(.10)$ & $.75(.07)$ & $.78(.07)$ & $.50(.10)$ & $.50(.09)$ \\
\hline
\end{tabular}

Note. $\quad$ n.a. $=$ not applicable.

${ }^{a}$ Highest loadings of each model shown in boldface, standard errors in brackets. 
model, $36 \%$ of the younger and $41 \%$ of the older adults had an RMSEA $<.05$.

A considerable group of persons showed no variance on the cardiovascular complaints variable. We reestimated the models without this variable, resulting in factor solutions for 75 younger adults (average RMSEA $=.07, S D=.09$, range $=.00-.29$ ), and for 52 older adults (average RMSEA $=.06, S D=.10$, range $=$ $.00-.36$ ). Of these, $61 \%$ of the younger and $67 \%$ of the older adults had an RMSEA $<.05$.

In sum, the one-factor solution had, on the one hand, an acceptable fit for a considerable number of persons. On the other hand, these analyses revealed a large degree of structural heterogeneity. The fit was not satisfactory for some persons, indicating that their individual within-person structures are more complex than a unidimensional one-factor solution, and for others the model could not be estimated in the first place because some of the complaints did not vary from day to day.

\section{Discussion}

This study investigated age differences in self-reported health symptoms with regard to mean levels, intraindividual variability, and within- versus between-person factor structures. In sum, there were small age differences in the means. Older adults fluctuated reliably less from day-to-day than did younger adults. The one-factor solution fit well at the average withinand between-person levels in both age groups. At the same time, reliable differences in loading patterns, particularly between the age groups, were observed. Furthermore, follow-up analyses at the individual level revealed that the one-factor model fails to provide an adequate rendition of day-to-day variation in self-reported health complaints for a substantial proportion of individuals in both age groups.

\section{Age Differences in Mean Levels}

We observed few age differences in means. Expected age differences favoring younger adults were found with a small effect size for cardiovascular complaints only. In contrast to our predictions, younger adults reported reliably more upper respiratory complaints, and especially more exhaustion than did older adults. This is not in line with previous research showing a decrease, albeit small, in subjective health across the adult life span (e.g., Staudinger et al., 1999; Wurm et al., 2009). However, these previous studies investigated global subjective health ratings and not day-to-day symptom reporting. On a day-to-day level, individuals may focus less on their general subjective health or objective health status, and report more minor symptoms that occur independently or in addition to chronic diseases. Self-reported complaints may also represent a physical well-being measure showing a similar trajectory over the life span as psychological well-being, namely relative stability (e.g., Costa et al., 1987). The well-being paradox (e.g., Staudinger, Marsiske, \& Baltes, 1995) may thus not only describe changes in psychological well-being measures, but also in self-reported physical well-being. Additionally, or alternatively, the observed age differences in average health complaints can also be interpreted from a cohort perspective. Younger adults reporting more exhaustion on a day-to-day level than older adults are probably leading a different lifestyle, with stress and subse- quent exhaustion being a part of it. Cardiovascular complaints are a typical health problem for older adults. It is possible that younger adults experience physical well-being primarily along a dimension of exhaustion. In line with this interpretation, exhaustion showed the highest within-person factor loading in younger adults (see Table 5). In contrast, older adults appeared to focus more on cardiovascular problems than younger adults.

\section{Age Difference in Intraindividual Variability}

The means of the health complaints were rather low in both age groups, which could have led to a smaller chance to detect intraindividual variability. Still, substantial intraindividual variability in subjective health complaints was found. In contrast to our predictions, older adults varied less from one day to the next. Several factors may contribute to the observed age-related reduction in the average day-to-day variability of subjective health. Relative to younger adults, lower variability of health-related complaints may reflect a dampening of situational effects operating on a day-to-day basis when objective health is low. Also, older adults may react less sensitively to daily events due to lifelong habituation processes or simply experience fewer events in a more tranquil lifestyle. As a fourth possible explanation, older adults may be more concerned about their health than younger adults, and may tend to answer health-related questions based on self-related judgments retrieved from long-term memory instead of actually probing their current health states. Such memory effects have been shown before for emotional self-reports (Robinson \& Clore, 2002), and may also apply to subjective reports of physical well-being. Additionally, personality characteristics, such as neuroticism, are assumed to be related to variability in mood and levels of health complaints. Thereby, they could be an explanation for the interindividual differences in fluctuations of self-reported health complaints. This may have contributed to the found age differences, as older adults are known to have lower scores in neuroticism than younger adults (e.g., Roberts, Walton, \& Viechtbauer, 2006). Future research is needed to explore which of these possible explanations applies, and how the amount of intraindividual variability and its change over the life span relates to other outcomes, such as objective health measures, mortality, or resiliency. First empirical evidence for the association with mortality already indicates that intraindividual variability in subjective health may be an important facet of the health status of a person (Ghisletta et al., 2002).

\section{Equivalence of Factor Structures Across Levels and Age Groups}

To test whether the between- and within-person structures in and across the age groups were equivalent, we estimated four submodels in one multigroup two-level confirmatory factor analysis: an average within-person model for YA, a between- person model for YA, an average within-person model for OA, and a between-person model for OA. The assumed one-factor solution fit well at the between-person level after allowing for a few correlated residuals, and also at the average within-person level.

The similarity of factor structures between the two age groups, and between between-person and average within-person structures was examined in three ways: (a) the RDR, (b) the overall $\chi^{2}$ 
difference test, and (c) differences in specific loadings. According to the RDR, setting the loadings equal across levels and age groups revealed an unsubstantial loss of fit for all but one of the tested models. Specifically, setting the within-person loadings to be equal across age groups resulted in a substantial loss of fit. According to the $\chi^{2}$ difference test, however, all models showed significant differences. On the one hand, this indicates that there are reliable differences between the factor structures in different age groups and at different levels, which motivates more detailed examinations of individual differences in within-person structures, as illustrated in the follow-up analyses. On the other hand, it should be noted that the power to detect even minor differences in factor loadings is high, with about 10,000 observations (Participants $\times$ Occasions) for each age group. The power analysis for the difference tests was estimated using the Monte Carlo simulation option with Mplus two-level models (see last column in Table 4), indicating high levels of observed power for the model comparisons. Evaluations of differences in structures should therefore not only be based on the significance of the model comparison, but also on considerations as regard content. The one-factor solution fit well at the between- and even better at the average within-person level, and configural invariance could be demonstrated for these health complaints across age groups and levels.

Post hoc comparisons of individual loadings revealed that the older adults' between-person structure showed the largest differences to the other submodels. Headaches and limb aches, not exhaustion as in the other models, was the most important variable to differentiate between older adults. The younger adults' factor loadings were very similar across levels; only restlessness represented individual differences better than differences within persons. Age differences were especially expressed in exhaustion and cardiovascular complaints. In accordance with the mean differences, exhaustion was an important variable to represent within- and between-person variation of younger adults, whereas cardiovascular complaints had higher loadings in the older adults' models. In sum, the differences in the loading patterns between age groups, especially concerning the older adults' between-person model, were more pronounced than differences between levels within the age groups. For older adults, more specific symptoms, like cardiovascular ones, seemed to be more relevant to describing their health status, whereas younger adults may best be described with overall exhaustion. This brings into focus that health, especially subjective health, may have different meanings for younger and older adults. With a normatively declining objective health status in old age, specific symptoms, potentially indicating an ongoing disease, may become more relevant than general exhaustion. In contrast, younger adults who have a better objective health status in general may describe their symptoms more likely as exhaustion. They may be less likely to interpret symptoms such as dizziness or poor circulation as indicators of serious cardiovascular complaints, but rather as less threatening overall exhaustion.

In follow-up analyses, we evaluated the one-factor structures at the individual level. The results showed an acceptable fit of the one-factor solution for a considerable number of persons. For others, the fit of the one-factor model was not satisfactory. Also, in many cases, the model could not be estimated in the first place because some of the complaints did not vary from day to day. This substantial heterogeneity suggests that complaints relate to physical well-being in a more or less idiosyncratic manner. One person is more likely to suffer from headaches, while another person reports more respiratory complaints. Because of the limited number of health complaints in the present study, an in-depth exploration of this heterogeneity is beyond the scope of this article. To come to a more detailed picture, the assessment of more complaints and a hierarchical structure of the complaints (e.g., several items that assess respiratory complaints) would be desirable. With such a measurement scheme, we would expect even larger differences between the between-person level and the average withinperson level, as well as between the factor solutions of each individual.

With these caveats in mind, it seems viable to investigate average within-person health complaints with a summary score across all complaints if a limited number of complaints is available. For example, if one is interested in day-to-day covariation between subjective health complaints and affective well-being in different age groups, the use of an aggregate score of health complaints may be justified.

\section{Limitations and Future Research Directions}

The generalizations from these results need to be made with caution. The older adult sample may well be fitter than the general population, as shown in comparisons with the representative Berlin subsample of the SOEP. Also, replications are needed to come to more generalizable results. In addition, comparisons between age groups are prone to cohort effects. Longitudinal studies are needed to investigate the development of symptom reporting and day-today fluctuation over the life span. As the older adults scheduled their sessions predominantly before noon and the younger adults later in the day, time of the day may have influenced the amount and strength of complaints reported. Some symptoms are stronger in the morning, others in the evening. Because we asked for symptoms at that particular day, there is more time to refer to in the afternoon sessions than in the morning sessions, which may have led to recollection biases. Also, participants who may have had severe symptoms caused by, for example, a flu, were of course less likely to come to the laboratory than healthy participants, which may have led to biases in the complaints reported.

As already mentioned, the health complaints used in this study were not exhaustive and were not assessed with several items per health domain. A broader representation of the health domains would be more likely to result in a more differentiated factor structure. The correlated residuals in the between-person models already indicate that a factor analysis with more complaints may result in other structures than the one-factor solution. Also, we excluded the gastrointestinal complaints item. For the older adults, the variability of this item consisted almost solely of within-person variability, and only of a small amount of between-person variability. For this particular item, the large proportion of withinperson variance would have been ignored if one had only studied the between-person level, underlining the importance of a withinperson approach. In future research, it will be interesting to see how variability as a facet of health contributes to the description of a person's health status, and examine its power to predict the development of health and other outcomes. Future research should also clarify the reasons for less variability in old age by, for example, investigating whether this lower level of fluctuation is mirrored in physiological health measures. 
We would like to highlight that the ergodicity assumption postulates not only the identity of between- and average within-person factor structures but also the identity of all within-person structures in the population (Molenaar, 2004; Molenaar \& Campbell, 2009). In the follow-up analyses, we found initial evidence that a substantial number of individuals was not well described by the one-factor model, which is clearly at odds with the ergodicity assumption. However, due to the restricted number of complaints, a sophisticated investigation of different factor structures for each individual was not conducted. In addition, the temporal dependency of the data is not modeled in Mplus two-level CFA, which might have influenced results to some degree. Person-centered analyses that also account for temporal dependencies, such as dynamic factor analysis, are needed to further investigate betweenperson differences in the within-person factor structures of health complaints. In contrast to such analyses, which require a large number of measurement occasions for each individual, two-level CFA is also applicable to data sets with a relatively small number of measurement occasions per person. Hence, we would like to encourage future research dealing with within-person relations to explicitly compare average within-person factor structures with between-person factor structures before interpreting the data at the individual level.

\section{Conclusion}

We showed that a one-factor solution of all health complaints fit the data well in both age groups at the average within-person level, for a considerable number of individuals, and after inclusion of a few correlated residual variances, also at the between-person level. Based on these results, the use of a summary score of health complaints for investigating within-person relationships in agecomparative studies appears justified. The present results do not permit the conclusion that the structure of the subjective health complaints is identical across all individuals. Given that individuals develop in unique ways (Nesselroade, 1991), we expect that future analyses with a more complex assessment of health status will reveal the heterogeneity of within-person structures.

Concerning the age group comparison, we demonstrated that there are few age differences in mean levels of symptoms reported on a day-to-day level. This finding is reminiscent of the well-being paradox (e.g., Staudinger et al., 1995), suggesting that subjective symptoms may be interpreted as a physical well-being measure. The health complaints seem to contribute differently to subjective health status in younger and older adulthood. According to the loading patterns and mean differences, younger adults seem to reflect their physical well-being more on an exhaustion dimension, whereas for older adults, cardiovascular complaints seem to play an important role. Concerning day-to-day variability, older adults fluctuated less in almost all assessed health symptoms, which may have different explanations. These results should encourage future research to investigate the causes for less variability in old age, and raise the question whether subjective health complaints are experienced and reported differently in younger and older adulthood.

\section{References}

Affleck, G., Tennen, H., Urrows, S., \& Higgins, P. (1994). Person and contextual features of daily stress reactivity: Individual differences in relations of undesirable daily events with mood disturbance and chronic pain. Journal of Personality and Social Psychology, 66, 329-340. doi: 10.1037/0022-3514.66.2.329

Aldwin, C. M., Park, C. L., \& Spiro, III, A. (2007). Health psychology and aging: An introduction. In C. M. Aldwin, C. L. Park, \& A. Spiro III (Eds.), Handbook of health psychology and aging (pp. 3-8). New York, NY: Guilford Press.

Becker, P., Schulz, P., \& Scholtz, W. (2004). Persönlichkeit, chronischer Stress und körperliche Gesundheit: Eine prospektive Studie zur Überprüfung eines systemischen Anforderungs-Ressourcen-Modells [Personality, chronic stress, and physical health: A prospective study to evaluate a systemic demand-resource model]. Zeitschrift für Gesundheitspsychologie, 12, 11-23. doi:10.1026/0943-8149.12.1.11

Benyamini, Y., \& Idler, E. L. (1999). Community studies reporting associations between self-rated health and mortality: Additional studies, 1995-1998. Research on Aging, 21, 392-401. doi:10.1177/ 0164027599213002

Borsboom, D., Mellenbergh, G., \& van Heerden, J. (2003). The theoretical status of latent variables. Psychological Review, 110, 203-219. doi: 10.1037/0033-295X.110.2.203

Brähler, E., Hinz, A., \& Scheer, J. W. (2008). Der Giessener Beschwerdebogen. Bern, Switzerland: Huber.

Brose, A., Schmiedek, F., Lövdén, M., \& Lindenberger, U. (2011). Normal aging dampens the link between intrusive thoughts and negative affect in reaction to daily stressors. Psychology and Aging, 26, 488-502. doi: 10.1037/a0022287

Brose, A., Schmiedek, F., Lövdén, M., Molenaar, P., \& Lindenberger, U. (2010). Adult age differences in covariation of motivation and working memory performance: Contrasting within- and between-person findings. Research in Human Development, 7, 61-78. doi:10.1080/ 15427600903578177

Browne, M., \& Du Toit, S. (1992). Automated fitting of nonstandard models. Multivariate Behavioral Research, 27, 269-300. doi:10.1207/ s15327906mbr2702_13

Charles, S. T., \& Almeida, D. M. (2006). Daily reports of symptoms and negative affect: Not all symptoms are the same. Psychology \& Health, 21, 1-17. doi:10.1080/14768320500129239

Costa, P. T. J., Zonderman, A. B., McCrea, R. R., Cornoni-Huntley, J., Locke, B. Z., \& Berbano, H. E. (1987). Longitudinal analysis of psychological well-being in a national sample: Stability of mean levels. Journal of Gerontology, 42, 50-55. doi:10.1093/geronj/42.1.50

Ghisletta, P., Nesselroade, J. R., Featherman, D. L., \& Rowe, J. W. (2002). Structure and predictive power of intraindividual variability in health and activity measures. Swiss Journal of Psychology, 61, 73-83. doi: 10.1024//1421-0185.61.2.73

Hamaker, E. L., Nesselroade, J. R., \& Molenaar, P. C. M. (2007). The integrated trait-state model. Journal of Research in Personality, 41, 295-315. doi:10.1016/j.jrp.2006.04.003

Idler, E. L., \& Benyamini, Y. (1997). Self-rated health and mortality: A review of twenty-seven community studies. Journal of Health and Social Behavior, 38, 21-37. doi:10.2307/2955359

MacDonald, S. W. S., Li, S.-C., \& Bäckman, L. (2009). Neural underpinnings of within-person variability in cognitive functioning. Psychology and Aging, 24, 792-808. doi:10.1037/a0017798

Molenaar, P. (2004). A manifesto on psychology as idiographic science: Bringing the person back into scientific psychology, this time forever Measurement: Interdisciplinary Research and Perspectives, 2, 201-218. doi:10.1207/s15366359mea0204_1

Molenaar, P., \& Campbell, C. G. (2009). The new person-specific paradigm in psychology. Current Directions in Psychological Science, 18, 112-117. doi:10.1111/j.1467-8721.2009.01619.x

Muthén, B. O. (1994). Multilevel covariance structure analysis. Sociological Methods Research, 22, 376-398. doi:10.1177/ 0049124194022003006 
Muthén, B. O., \& Satorra, A. (1995). Complex sample data in structural equation modeling. Sociological Methodology, 25, 267-316. doi: $10.2307 / 271070$

Nesselroade, J. R. (1991). The warp and the woof of the developmental fabric. In R. M. Downs, L. S. Liben, \& D. S. Palermo (Eds.), Visions of aesthetics, the environment \& development: The legacy of Joachim F. Wohlwill (pp. 213-240). Hillsdale, NJ: Erlbaum.

Nesselroade, J. R. (2001). Intraindividual variability in development within and between individuals. European Psychologist, 6, 187-193. doi: 10.1027/1016-9040.6.3.187

Ong, A. D., \& Allaire, J. C. (2005). Cardiovascular intraindividual variability in later life: The influence of social connectedness and positive emotions. Psychology and Aging, 20, 476-485. doi:10.1037/08827974.20.3.476

Roberts, B. W., Walton, K. E., \& Viechtbauer, W. (2006). Patterns of mean-level change in personality traits across the life course: A metaanalysis of longitudinal studies. Psychological Bulletin, 132, 1-25. doi: 10.1037/0033-2909.132.1.1

Robinson, M. D., \& Clore, G. L. (2002). Episodic and semantic knowledge in emotional self-report: Evidence for two judgment processes. Journal of Personality and Social Psychology, 83, 198-215. doi:10.1037/00223514.83.1.198

Schmiedek, F., Bauer, C., Lövdén, M., Brose, A., \& Lindenberger, U. (2010). Cognitive enrichment in old age: Web-based training programs. GeroPsych: Journal of Gerontopsychology and Geriatric Psychiatry, 23, 59-67. doi:10.1024/1662-9647/a000013

Schmiedek, F., Lövdén, M., \& Lindenberger, U. (2009). On the relation of mean reaction time and intraindividual reaction time variability. Psychology and Aging, 24, 841-857. doi:10.1037/a0017799
Schmiedek, F., Lövdén, M., \& Lindenberger, U. (2010). Hundred days of cognitive training enhanced broad cognitive abilities in adulthood: Findings from the COGITO Study. Frontiers in Aging Neuroscience, 2, 27. Online publication. doi:10.3389/fnagi.2010.00027

Schwartz, G. L., Tuner, S., Moore, J., \& Sing, C. (2000). Effect of time of day on intraindividual variability in ambulatory blood pressure. American Journal of Hypertension, 13, 1203-1209. doi:10.1016/S08957061(00)01198-5

Staudinger, U., Fleeson, W., \& Baltes, P. B. (1999). Predictors of subjective physical health and global well-being: Similarities and differences between the United States and Germany. Journal of Personality and Social Psychology, 76, 305-319. doi:10.1037/0022-3514.76.2.305

Staudinger, U. M., Marsiske, M., \& Baltes, P. B. (1995). Resilience and reserve capacity in later adulthood: Potentials and limits of development across the life span. In C. Cicchetti, \& D. J. Cohen (Eds.), Developmental Psychopathology (pp. 801-847). New York, NY: Wiley.

Wagner, G. G., Frick, J. R., \& Schupp, J. (2007). The German SocioEconomic Panel Study (SOEP): Scope, evolution and enhancements. Schmollers Jahrbuch, 127, 139-169.

Wurm, S., Lampert, T., \& Menning, S. (2009). Subjektive Gesundheit [Subjective health]. In K. Böhm, C. Tesch-Römer, \& T. Ziese (Eds.), Gesundheit und Krankheit im Alter (pp. 79-91). Berlin, Germany: Robert Koch-Institut.

Received July 1, 2011

Revision received April 20, 2012

Accepted April 23, 2012

\section{Correction to Price and Murray (2012)}

In the article "The Region of Proximal Learning Heuristic and Adult Age Differences in SelfRegulated Learning," by Jodi Price and Rory G. Murray (Psychology and Aging, Advance online publication. September 3, 2012. doi:10.1037/a0029860), there is an error on page 8, in the paragraph "Item selection."

The second sentence in the paragraph should read:

"The MCI-Potential Improvement scale also predicted the proportion of medium items studied $(\beta=-.19, t=-2.20, p<.05), F(2,110)=15.40, p<.001, R^{2}=.22$, and the proportion of complex items studied, $(\beta=-.19, t=-2.25, p<.05), F(2,110)=14.48, p<.001, R^{2}=.21$." 